\title{
The Uncertainty in Newton's Constant and Precision Predictions of the Primordial Helium Abundance
}

\author{
Robert J. Scherrer \\ Department of Physics and Astronomy, Vanderbilt University, Nashville, TN 37235
}

\begin{abstract}
The current uncertainty in Newton's constant, $G_{N}$, is of the order of $0.15 \%$. For values of the baryon to photon ratio consistent with both cosmic microwave background observations and the primordial deuterium abundance, this uncertainty in $G_{N}$ corresponds to an uncertainty in the primordial ${ }^{4} \mathrm{He}$ mass fraction, $Y_{P}$, of $\pm 1.3 \times 10^{-4}$. This uncertainty in $Y_{P}$ is comparable to the effect from the current uncertainty in the neutron lifetime, $\tau_{n}$, which is often treated as the dominant uncertainty in calculations of $Y_{P}$. Recent measurements of $G_{N}$ seem to be converging within a smaller range; a reduction in the estimated error on $G_{N}$ by a factor of 10 would essentially eliminate it as a source of uncertainty the calculation of the primordial ${ }^{4} \mathrm{He}$ abundance.
\end{abstract}

Big Bang nucleosynthesis (BBN) represents one of the key successes of modern cosmology [1, 2]. In recent years, the BBN production of deuterium has emerged as the most useful constraint on the baryon density in the universe, both because of observations of deuterium in (presumably unprocessed) high-redshift QSO absorption line systems (see Ref. [3], and references therein), and the fact that the predicted BBN yields of deuterium are highly sensitive to the baryon density. These arguments give a baryon density parameter $\Omega_{b} h^{2}$, of [3]

$$
\Omega_{b} h^{2}=0.0194-0.0234,
$$

which is in excellent agreement with the baryon density derived by the WMAP team from recent cosmic microwave background observations [4]:

$$
\Omega_{b} h^{2}=0.022-0.024 \text {. }
$$

Given these limits on the baryon density, BBN predicts the primordial abundances of ${ }^{4} \mathrm{He}$ and ${ }^{7} \mathrm{Li}$. Because the ${ }^{4} \mathrm{He}$ abundance is particularly sensitive to new physics beyond the standard model, a comparison between the predicted and observed abundances of ${ }^{4} \mathrm{He}$ can be used to constrain, for example, neutrino degeneracy or extra relativistic degrees of freedom (see, e.g., Refs. [5, 6]).

For this reason, it is useful to obtain the most accurate possible theoretical predictions for the primordial ${ }^{4} \mathrm{He}$ mass fraction, $Y_{P}$. The primordial production of ${ }^{4} \mathrm{He}$ is controlled by the competition between the rates for the processes which govern the interconversion of neutrons and protons,

$$
\begin{aligned}
n+\nu_{e} & \leftrightarrow p+e^{-} \\
n+e^{+} & \leftrightarrow p+\bar{\nu}_{e} \\
n & \leftrightarrow p+e^{-}+\bar{\nu}_{e}
\end{aligned}
$$

and the expansion rate of the Universe, given by

$$
\frac{\dot{R}}{R}=\left(\frac{8}{3} \pi G_{N} \rho\right)^{1 / 2} .
$$

In BBN calculations, the weak interaction rates are scaled off of the inverse of the neutron lifetime, $\tau_{n}$.
When these rates are faster than the expansion rate, the neutron-to-proton ratio $(n / p)$ tracks its equilibrium value. As the Universe expands and cools, the expansion rate comes to dominate and $n / p$ essentially freezes out. Nearly all the neutrons which survive this freezeout are bound into ${ }^{4} \mathrm{He}$ when deuterium becomes stable against photodisintegration. Following the initial calculations of Wagoner, Fowler, and Hoyle 7], numerous groups examined higher-order corrections to the ${ }^{4} \mathrm{He}$ production. The first such systematic attempt was undertaken by Dicus et al. [8], who examined the effects of Coulomb and radiative corrections to the weak rates, finite-temperature QED effects, and incomplete neutrino coupling. Later investigations included more detailed examination of Coulomb and radiative corrections to the weak rates 9, 10, 11, 12, finite-temperature QED effects 13], and incomplete neutrino decoupling [14], as well as an examination of the effects of finite nuclear mass 15. These effects were systematized by Lopez and Turner 16] (see also Ref. 17]), who argued that all theoretical corrections larger than the effect of the uncertainty in the neutron lifetime had been accounted for, yielding a total theoretical uncertainty $\Delta Y_{P}<0.0002$. Assuming an uncertainty of \pm 2 sec in the neutron lifetime, the corresponding experimental uncertainty in $Y_{P}$ is $\Delta Y_{P}= \pm 0.0004$. Similar results were obtained in Ref. [17.

Two relevant changes have occurred since the publication of Refs. 16, 17]. First, the estimated uncertainty in the neutron lifetime has decreased, with the current value being [18]

$$
\tau_{n}=885.7 \pm 0.8 \mathrm{sec}
$$

Second, the estimated uncertainty in the value of $G_{N}$ has increased. The value currently recommended by CODATA (Committee on Data for Science and Technology) is 19

$$
G_{N}=6.673 \pm 0.010 \times 10^{-8} \mathrm{~cm}^{3} \mathrm{gm}^{-1} \mathrm{sec}^{-2} .
$$

This represents a factor of twelve increase over the previous recommended uncertainty [20], and is primarily due to an anomalously high value for $G$ determined by Michaelis, Haars, and Augustin [21]. (See Table 1). 


\begin{tabular}{|l|l|}
\hline \hline$G_{N} \cdot 10^{8}\left[\mathrm{~cm}^{3} \mathrm{~g}^{-1} \mathrm{sec}^{-2}\right]$ & Reference \\
\hline \hline $6.67407(22)$ & Schlamminger et al. (2002) [29] \\
$6.67559(27)$ & Quinn, et al. (2001) [30] \\
$6.674215(92)$ & Gundlach and Merkowitz (2000) [31] \\
$6.6699(7)$ & Luo et al. (1999) [32] \\
$6.6742(7)$ & Fitzgerald and Armstrong (1999) [33] \\
$6.6873(94)$ & Schwarz et al. (1999) [34] \\
$6.6749(14)$ & Nolting, et al. (1999) [35] \\
$6.6735(29)$ & Kleinevoss et al. (1999) [36] \\
$6.673(10)$ & CODATA (1998) [19] \\
$6.67259(85)$ & CODATA (1986) [20] \\
\hline \hline
\end{tabular}

TABLE I: Recent experimental values of Newton's constant. Digits in parentheses are the 1- $\sigma$ uncertainty in the last digits of the given value.

It is easy to calculate the effect of both of these uncertainties on the primordial ${ }^{4} \mathrm{He}$ abundance. For the range of values of $\Omega_{b} h^{2}$ given in equations (1) and (2), we find, numerically,

$$
\begin{aligned}
& \Delta Y_{P}=0.088\left(\Delta G_{N} / G_{N}\right) \\
& \Delta Y_{P}=0.18\left(\Delta \tau_{n} / \tau_{n}\right) .
\end{aligned}
$$

The coefficient in equation (8) is twice that in equation (7). This factor of 2 comes from the fact that the expansion rate (equation 4 ) scales as $G_{N}^{1 / 2}$, while the weak interaction rates scale as $\tau_{n}^{-1}$, and the abundance of ${ }^{4} \mathrm{He}$ is essentially unchanged if the ratio of the weak interaction rates to the expansion rate is held constant.

Then the current uncertainties in $G_{N}$ and $\tau_{n}$ given in equations (5) and (6) yield, for the $1-\sigma$ uncertainties in $Y_{P}$

$$
\Delta Y_{P}= \pm 1.6 \times 10^{-4}
$$

from the uncertainty in $\tau_{n}$, and

$$
\Delta Y_{P}= \pm 1.3 \times 10^{-4}
$$

from the uncertainty in $G_{N}$.

These two uncertainties are roughly comparable. This is significant because the uncertainty in $\tau_{n}$ is often taken to be the dominant uncertainty in calculations of $Y_{P}$. Of course, both of these effects are exceedingly small, and well below the dispersion in the estimates of the primordial ${ }^{4} \mathrm{He}$ abundance from observations of low-metallicity systems [2]. (The effect of the uncertainty in $G_{N}$ is comparable to the corrections to $Y_{P}$ due to QED plasma effects and residual neutrino heating [16]). A further source of uncertainty in theoretical calculations of $Y_{P}$ is the uncertainty in the nuclear reaction rates. A recent exhaustive study of this effect has been undertaken by Cyburt 22], who concluded that the effect on $Y_{P}$ of the uncertainties in the nuclear reaction rates is currently subdominant. (See also earlier work in Refs. 23, 24]).

The uncertainty in $G_{N}$ has consequences in other astrophysical settings. Lopes and Silk 25] investigated the effect on the sound speed in the sun. They argued that helioseismology (in combination with improved solar neutrino measurements) might eventually provide an independent constraint on $G_{N}$, although this claim has been disputed by Ricci and Villante [26].

In principle, the uncertainty in $G_{N}$ also affects CMB measurements. The change in the observed CMB fluctuation spectrum due to a fixed change in $G_{N}$ was investigated by Zahn and Zaldarriaga 27]. Even under the most optimistic conditions for future observations, the smallest change in $G_{N}$ which is, in principle, detectable in CMB measurements is $\Delta G_{N} / G_{N} \sim 0.006$, well above the current CODATA uncertainty.

It is likely, of course, that current and future measurements will lead to a reduction in the uncertainty in $G_{N}$. A set of the most recent measurements of $G_{N}$ is displayed in Table 1. (For a survey of measurements of $G_{N}$ over a longer timeline, see Ref. [28]). The three most recent measurements all yield a value of $G_{N}$ within a very narrow range. Reduction in the uncertainty in $G_{N}$ by, for example, a factor of 10 (e.g., back to the 1986 CODATA level of uncertainty) would essentially eliminate any significant effect on BBN calculations.

\section{Acknowledgments}

I thank M. Kaplinghat and G. Greene for helpful discussions.
[1] D.N. Schramm and M.S. Turner, Rev. Mod. Phys. 70, 303 (1998).

[2] K.A. Olive, G. Steigman, and T.P. Walker, Phys. Rep. 333, 389 (2000).

[3] D. Kirkman, D. Tytler, N. Suzuki, J.M. O'Meara, and D. Lubin, astro-ph/0302006

[4] D. Spergel, et al., Ap.J. Suppl. 148, 175 (2003).

[5] J.P. Kneller, R.J. Scherrer, G. Steigman, and T.P. Walker, Phys. Rev. D 64, 123506 (2001).

[6] V. Barger, J.P. Kneller, H.-S. Lee, D. Marfatia, and G.
Steigman, Phys. Lett. B 566, 8 (2003).

[7] R.V. Wagoner, W.A. Fowler, and F. Hoyle, Ap.J. 148, 3 (1967).

[8] D.A. Dicus, et al, Phys. Rev. D 26, 2694 (1982).

[9] J.F. Donoghue and B.R. Holstein, Phys. Rev. D 28, 340 (1983).

[10] J.F. Donoghue and B.R. Holstein, Phys. Rev. D 29, 3004 (1984).

[11] R.F. Sawyer, Phys. Rev. D 53, 4232 (1996).

[12] I.A. Chapman, Phys. Rev. D 55, 6287 (1997). 
[13] A.J. Heckler, Phys. Rev. D 49, 611 (1994).

[14] S. Dodelson and M.S. Turner, Phys. Rev. D 46, 3372 (1992).

[15] R.E. Lopez, M.S. Turner, and G. Gyuk, Phys. Rev. D 56, 3191 (1997).

[16] R.E. Lopez and M.S. Turner, Phys. Rev. D 59, 103502 (1999).

[17] S. Esposito, G. Mangano, G. Miele, and O. Pisanti, Nucl. Phys. B540, 3 (1999).

[18] K. Hagiwara, et al., Phys. Rev. D 66, 010001 (2002).

[19] P.J. Mohr and B.N. Taylor, Rev. Mod. Phys. 72, 351 (2000).

[20] E.R. Cohen and B.N. Taylor, Rev. Mod. Phys. 59, 1121 (1987).

[21] W. Michaelis, H. Haars, and R. Augustin, Metrologia 32, 267 (1996).

[22] R.H. Cyburt, astro-ph/0401091

[23] L.M. Krauss and P. Romanelli, Ap.J. 358, 47 (1990).

[24] L.M. Krauss and P. Kernan, Phys. Lett. B347, 347 (1995).

[25] I.P. Lopes and J. Silk, astro-ph/0112310
[26] B. Ricci and F.L. Villante, Phys. Lett. B 549, 20 (2002).

[27] O. Zahn and M. Zaldarriaga, Phys. Rev. D 67, 063002 (2003).

[28] G.T. Gillies, Meas. Sci. Technol. 10, 421 (1999).

[29] St. Schlamminger, E. Holzschuh, and W. Küdig, Phys. Rev. Lett. 89, 161102 (2002).

[30] T.J. Quinn, C.C. Speake, S.J. Richman, R.S. Davis, and A. Picard, Phys. Rev. Lett. 87, 111101 (2001).

[31] J.H. Gundlach and S.M. Merkowitz, Phys. Rev. Lett. 85, 2869 (2000).

[32] Luo, J., Hu, Z.K., Fu X.H., Fan S.H., and Tang, M.X., Phys. Rev. D 59, 042001 (1999).

[33] M.P. Fitzgerald and T.R. Armstrong, Meas. Sci. Technol 10, 439 (1999).

[34] J.P. Schwarz, D.S. Robertson, T.M. Niebauer, and J.E. Faller, Meas. Sci. Technol. 10, 478 (1999).

[35] F. Nolting, J. Schurr, S. Schlamminger, and W. Kündig, Meas. Sci. Technol. 10, 487 (1999).

[36] U. Kleinevoss, H. Meyer, A. Schumacher, and S. Hartmann, Meas. Sci. Technol. 10, 492 (1999). 